\title{
The change in the efficiency factor of the pumping unit with a frequency converter
}

\author{
Eduard Kan ${ }^{1,2, *}$, Nazir Ikramov ${ }^{1}$ and Muradulla Mukhammadiev ${ }^{2}$ \\ ${ }^{1}$ Tashkent Institute of Irrigation and Agricultural Mechanization Engineers, Department of \\ hydraulic construction, 100000 Tashkent, Uzbekistan \\ ${ }^{2}$ Tashkent State Technic University named after Islam Karimov , Department of power, 100095 \\ Tashkent, Uzbekistan
}

\begin{abstract}
This paper gives the results of the investigation of the operation of a pumping unit equipped with a frequency converter. As a rule, it is widely assumed that when changing the speed up to $50 \%$, the pump efficiency almost does not change, and the possibility of decrease of its efficiency is not taken into consideration when calculating the economic efficiency of the operation of the pumping unit equipped with a frequency converter. However, failure to take account the efficiency decrease may cause inaccurate assessment of the economic efficiency of the use of the frequency converter and, consequently, improper selection of the method of control of the operation of the pumping units. This is particularly important for irrigation pumping plants the operation of which is characterized by large volume of the pumpable volume and less severe requirements for provision of the water pressure required for the consumer. The purpose of this paper is to carry out experiments on a testbed equipped with a pumping unit, parameter measuring instrument, and a frequency converter, and detection of the pattern of efficiency variation with speed. The paper presents the results of the carried out experiments, the obtained characteristic curves of the variation of different types of the efficiency at variation of the pump shaft speed, as well as their analysis.
\end{abstract}

\section{Introduction}

When operating pumping plants, one will have to frequently change their parameters, i.e. rely on their control. There are a number of different control methods, however controlling by varying its impeller speed is considered to be the most economical one [1-8].

Electric motor speed control can be performed by using the following devices:

1.Mechanical variable-speed drive;

2.Electromagnetic or fluid-actuated clutch;

3.Multiple-speedmotor;

\footnotetext{
* Corresponding author: Kan_E1969@mail.ru
} 
4.Use of reduction gears or belt drives;

5.Frequency converter.

The first four methods have perceptible shortcomings: complexity of the structural design, application, operation and maintenance of these facilities; narrow range of control, and their economic in efficiency.

Therefore, in recent times the application of the method of pump impeller shaft speed variation by using a frequency converter is proposed as one of the efficient way of control of pump operation [9-13].

Frequency converters allow more accurate and full meeting of the pumping plant waterconsumption schedule, which will result in the reduction of non-productive water losses, and enable making smooth start and stop of the pumps. Even having such advantages, they do not take into account the possibility of loss of the pumping unit efficiency when calculating the economic efficiency of the use of frequency converters. Moreover, irrigation pumping plants differ from water supplying pumping plants in terms of the requirements set before them $[1-2,4,14]$. While the main requirements set before the latter are associated with the water supply and pressure (required hydraulic head in the water supply network), the main requirement set before irrigation pumping plants is to supply required water quantity during certain periods. Therefore, the pumping unit switching on and off control methods are widely applied in irrigation pumping plants (in particular, in our Republic). At that, pumping units operate with rated parameters at maximum efficiency factor, but for short time, and this operation is accompanied by frequent starts and stops. When using frequency converters, no frequent starts and stops will take place; however, the pump units will not run in the design energy conditions. The existing methods of the assessment of the economic efficiency of the frequency converter usage generally compare the effect of the frequency converter usage with the throttling method (control by means of the valve on the discharge line) $[9,10-13,15]$. However, the throttling method is not recommended to be applied at irrigation pumping plants, and it is almost no used [1, 2, 4].

Since the electric power costs account for the most part of the operating and maintenance costs of pumping plants with electrically driven motors, the issue of the quantitative evaluation of the efficiency decrease when frequency converters are used at irrigation pumping plants becomes topical.

\section{Methods}

The efficiency factor, i.e. ratio of the hydraulic energy received by the fluid to the mechanical energy applied to the shaft is a most significant parameter of the pump proceeding from which one can judge of its energy performance. It is generally believed that when varying the speed by less than $50 \%$, the volumetric and hydraulic efficiency does not change, and it is recommended to assumed constant when carrying out relevant calculations [1-8]. However, the operation has shown that in practice efficiency changes even at lower variations in the speed $[11,13,15,19]$. This is evidenced by the results of various field investigations carried out in this sphere in recent times $[13,19]$. The purpose of the laboratory investigations was to study the effect of a change in the pump shaft speed on different efficiency factors of the pump and the pumping unit as a whole. At that, one should bear in mind that the pump efficiency depends first of all on the pump size and then on the shape of the pump wet end, impeller, and structural design. Therefore, the obtained relationships are true right for the pump being tested and can be applied for full-sized pumps only of this type, i.e. geometrically similar pumps.

Experiments were conducted on a special test-bed equipped with a frequency converter in order to define the efficiency behavior.

The test-bed is composed of: 
1) single-stage horizontal centrifugal pump of CEA 70/33 (Lowara) type supplied with power from three-phase AC mains with a voltage of $220 \mathrm{~V}$;

2) control and measuring instruments:

-for measuring the pump inlet and outlet pressure: piezoresistive pressure sensor with a tensoresistive sensing element having the metallic measuring diaphragm and the built-in electronics. The measurement error of the device is $0.5 \%$.

- for measuring the pump capacity: an electromagnetic flowmeter "Vzlyot-ER" (in Russian: ВЗЛЕТ-ЭР). The measurement error is $\pm 2.0 \%$.

3) frequency converter "Danfoss"of VLT Micro Drive fc 051series.

The frequency converter operates based on the double-conversion circuit. It is composed of the following parts: DC link (uncontrolled rectifier), power pulse inverter, and control system. The DC link has an uncontrolled rectifier and a filter. In it, the AC voltage of the mains will be converted to DC voltage. The three-phase power pulse inverter consists of six transistor switches. Each electric motor winding is connected through the relevant switch to the negative or positive terminal of the rectifier. The inverter will convert the rectified voltage to three-phase voltage of the required frequency and amplitude, which is to be applied to the electric motor stator windings.

The frequency converter is composed of an uncontrolled diode power rectifier, selfexcited inverter, pulse-width modulator control system, and control panel.

The procedure for experiments is as follows: after starting the pump, its speed shall be controlled by means of the frequency converter control panel, and in case of any variation, they record the values of delivery rate and pressure difference at the pump upstream and downstream, and the electric motor power consumption. In total, 75 experiments have been conducted.

\section{Results}

The relationships of the change of different efficiency factors with variation of the pump shaft speed have been defined following the results of the experiments.

The efficiency of the pumping unit (a pump connected to a motor) comes to [1$8,14,20,21]$ :

$$
\eta_{\mathrm{u}}=\eta_{\mathrm{p}} \eta_{\mathrm{m}} \eta_{\text {transm }}
$$

where:

$\eta_{\mathrm{u}}$-is pumping unit efficiency;

$\eta_{\mathrm{p}}$-is pump efficiency;

$\eta_{\mathrm{m}}$-is driven motor efficiency;

$\eta_{\text {transm }}$-is transmission efficiency. In our case (monoblock type connection), $\eta_{\text {transm }}=1$.

As is well known, the pump efficiency is the ratio of the useful power to the pump power. The pump efficiency takes into account the hydraulic, volumetric, and mechanical losses arising when transmitting the energy of the pumped fluid and is equal to the product of the hydraulic, volumetric, and mechanical efficiency factors:

$$
\eta_{\mathrm{p}}=\eta_{\mathrm{h}} \eta_{\mathrm{v}} \eta_{\mathrm{m}}
$$

The volumetric losses include the losses due to the leakages through impeller seals and/or packing glands. These losses represent gap losses which are unavoidable. In 
centrifugal pumps, gap losses occur in the impeller seals between the discharge and suction zones:

$$
\eta_{\mathrm{v}}=\frac{\mathrm{Q}}{\mathrm{Q}+\mathrm{q}}
$$

where:

$\eta_{\mathrm{v}}$-is volumetric efficiency;

$Q$ - is pump delivery rate;

$q$ - is volumetric losses;

A change in the volumetric efficiency with a change of the impeller speed can be defined by using the results of the experts and the proportionality laws according to which the delivery rate varies $[7,14]$ :

$$
\frac{\mathrm{Q}}{\mathrm{Q}_{1}}=\left(\frac{\mathrm{n}}{\mathrm{n}_{1}}\right) *\left(\frac{\eta_{\mathrm{v}}}{\eta_{\mathrm{v} 1}}\right)
$$

$\mathrm{Q}, \eta_{\mathrm{v}}$ - are respectively the pump delivery rate and volumetric efficiency factor at speed $n$; $\mathrm{Q}_{1}, \eta_{\mathrm{v} 1}$ - are respectively the pump delivery rate and volumetric efficiency factor at speed $n_{l}$.

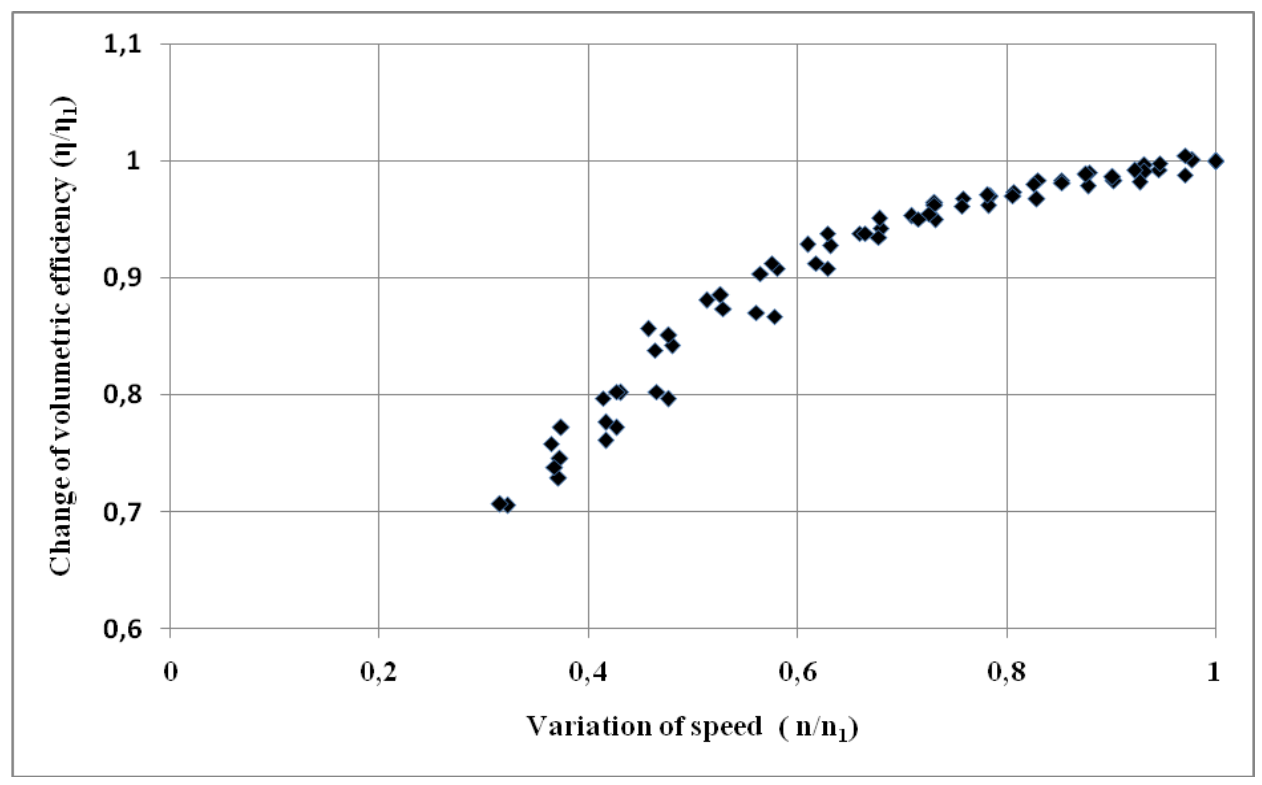

Fig. 1. Curve of the volumetric efficiency $\left(\eta_{v} / \eta_{v 1}\right)$ with variation of speed $\left(n / n_{1}\right)$.

To assess and compare the changes of the different efficiency factors of the pump $\left(\eta_{\mathrm{v}} / \eta_{\mathrm{v} 1}\right)$ with variation of the pump speed, we use relative indices. In such a case, all the values shall be determined with reference to the basic values (at a rated speed and maximum efficiency). Based on the results of the conducted experiments, the characteristic curve of the changes in the volumetric efficiency $\left(\eta_{\mathrm{v}} / \eta_{\mathrm{v} 1}\right)$ with variation of the speed 
$\left(\mathrm{n} / \mathrm{n}_{1}\right)$. The majority of the authors $[1-6,8,20,21]$ share the same opinion that the efficiency change is negligible and $\eta_{\mathrm{v}}=\eta_{\mathrm{v} 1}$. However, according to the results of the conducted experiments, these changes may be considerable enough.

Similarly, one can plot the characteristic curve of the changes in the hydraulic losses with speed variation. Hydraulic losses mean the total losses occurred during the fluid flow through the pump wet end channels and the churning losses due to flow separation when passing around various operating elements of the pump:

$$
\eta_{\mathrm{h}}=\frac{\dot{\mathrm{I}}}{\hat{\mathrm{I}}+\Delta \hat{\mathrm{I}}}
$$

where,

$\eta_{\mathrm{h}}$ - is the hydraulic efficiency of the pump;

$\mathrm{H}$ - is the head developed by the pump;

$\Delta \mathrm{H}^{-}$is the head losses in the pump.

Head will change with variation of its impeller speed according to the following similarity law [9]:

$$
\frac{\mathrm{H}}{\mathrm{H}_{1}}=\left(\frac{\mathrm{n}}{\mathrm{n}_{1}}\right)^{2} *\left(\frac{\eta_{\mathrm{h}}}{\eta_{\mathrm{h} 1}}\right)
$$

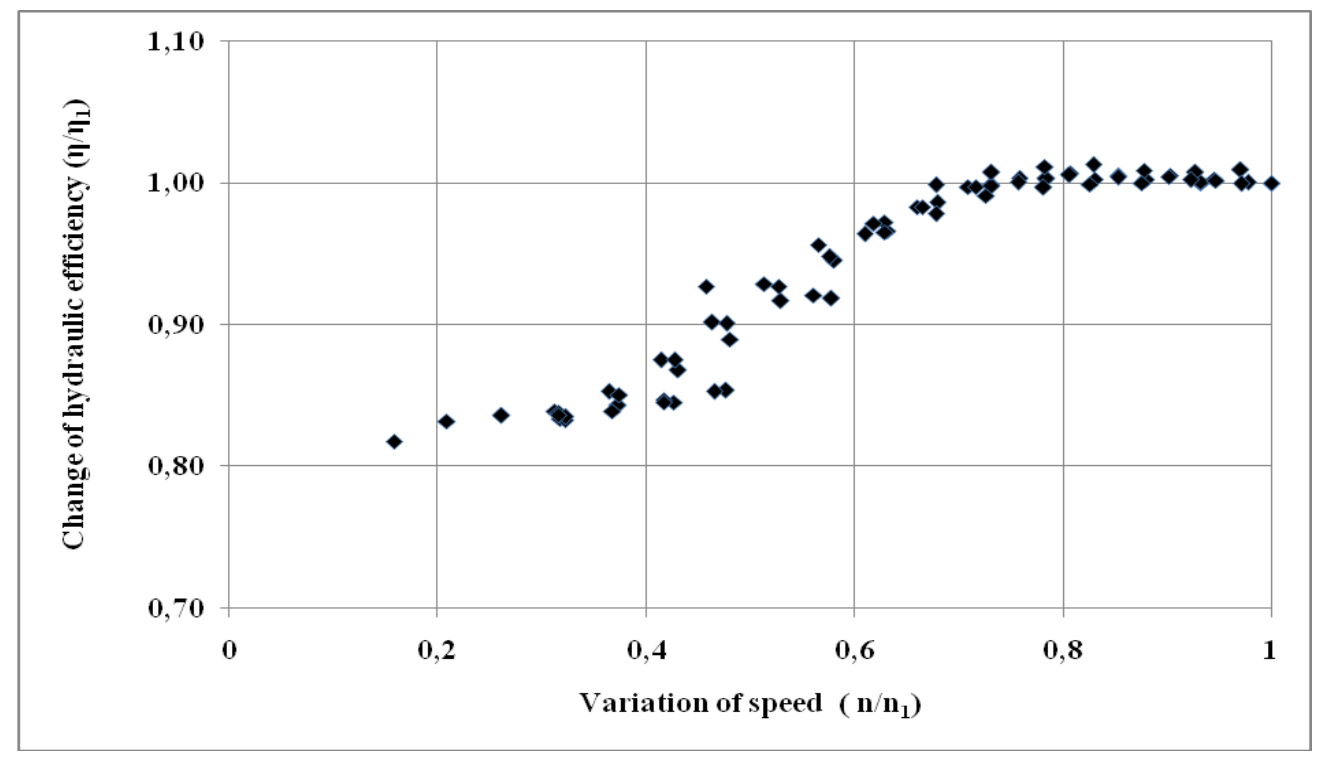

Fig. 2. Curve of the hydraulic efficiency $\left(\eta_{h} / \eta_{h 1}\right)$ with variation of speed $\left(n / n_{1}\right)$.

The efficiency of the pumping unit, i.e. a pump connected to a motor, is given by [18,14,20-21]:

$$
\eta_{\mathrm{u}}=\frac{\mathrm{N}_{\mathrm{us}}}{\mathrm{N}_{\mathrm{m}}}
$$


where

$\mathrm{N}_{\mathrm{us}}$ - is the useful power;

$\mathrm{N}_{\mathrm{m}}$ - is the pumping unit power or electric motor power.

The useful power can be determined by the following well-known formula: $N_{i}=\gamma Q H$, while the_electric motor power is known from the results of the conducted experiments. The_relationship of changes in the pumping unit efficiency $\left(\eta_{\mathrm{u}} / \eta_{\mathrm{u} 1}\right)$ with variation of the speed $\left(n / n_{1}\right)$ is shown in Fig. 3.

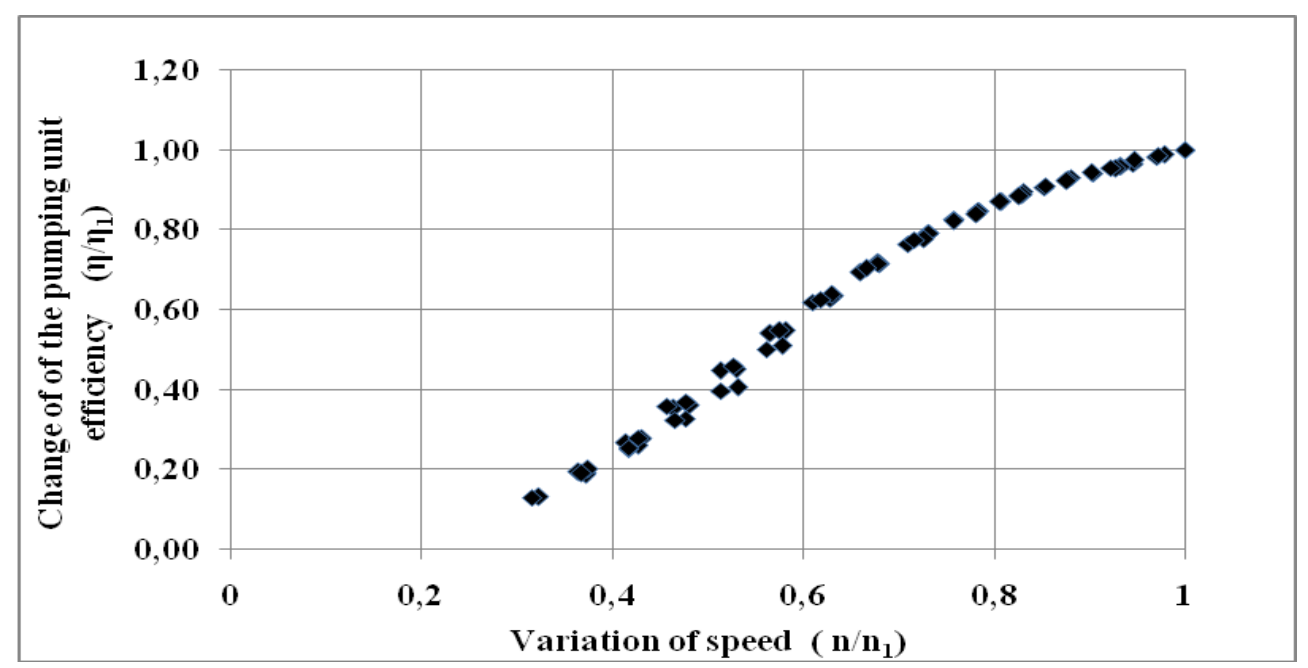

Fig. 3. Curve of the pumping unit efficiency $\left(\eta_{u} / \eta_{u 1}\right)$ with variation of speed $\left(n / n_{1}\right)$.

\section{Discussions}

As the pump impeller shaft speed decreases, the volumetic efficiency of the pump decreases too. For the CEA 70/33 (Lowara) type pump, the most intense decrease will take place with a speed decrease by more than $20 \%$ of the rated value (Fig. 1).

From the curve the hydraulic efficiency change with variation of speed presented in Fig. 2 it is obvious that the correlation curve has three characteristics zones:

- Zone 1 refers to the speed variation range (approximately up to 30\%), where the hydraulic efficiency almost does not change;

- Zone 2 (speed variation range from 30 to $65 \%$, i.e. $0.30<\mathrm{n} / \mathrm{n}_{1}<0.65$ ), characterized by intense decrease in the hydraulic efficiency;

- Zone 3 falls within the range of shaft speed variation by more than $65 \%$, where the hydraulic efficiency variation is insignificant.

In other words, the most intense change in the hydraulic efficiency will take place when the speed changes by more than $30 \%$ and less than $65 \%$. So, the authors' recommendations [1-3] that the hydraulic and volumetric losses will scarcely change with the speed variation up to $50 \%$ are only partly true. Indeed, the hydraulic efficiency will almost not change with speed variation up to $30 \%$. The chart of the pumping unit efficiency change as a whole (including the losses in the electric motor and mechanical losses in the pump in addition to the hydraulic and volumetric losses) depending on the drive shaft speed variation is shown in Fig. 3. Analysis of this characteristic curve shows 
that the pumping unit efficiency will continuously decline with the speed decrease from its rated value. For the CEA 70/33 (Lowara) type pump, when reducing the speed from the rated value of $2790 \mathrm{rpm}$ to $1390 \mathrm{rpm}$, the efficiency will drop by $65 \%$ of the rated efficiency factor. The fact of considerable decline in the pumping unit efficiency with a decrease in the pump shaft speed is confirmed by the results of the field experiments carried out at the pumping plant "Teshiktash-1" in the Andijan region [19].

\section{Conclusions}

A series of experiments have been conducted with a pumping unit equipped with a frequency converter for the purpose of defining the effect of speed variation on the parameters of the pumping unit. The conducted experimental investigations have shown the following: even a slight change in the impeller speed (considerably less than by 50\%) will cause changes in the pump efficiency. Different pump efficiency types change to different extents with lowering the speed. For example, if the CEA 70/33 (LOWARA) type pump shaft speed varies by $50 \%$, the hydraulic efficiency will change by $5-7 \%$, and the volumetric efficiency will decrease by $30 \%$, while the total efficiency of the pumping unit will drop by $65 \%$.

The results obtained show that when selecting a method of control of an irrigation pumping plant, one should take into consideration that if the speed of the pumping units change, they will not operate in the design operating conditions (with rated parameters) and considerable efficiency drop may take place at that.

When comparing different control methods for the irrigation pumping plant, the method of pumping unit "switching on \& switching off " method should be alternatively applied.

We would like to express our gratitude to the Center for Professional Advancement under the Tashkent Institute of Irrigation and Agricultural Mechanization Engineers for giving us an opportunity to conduct the experiments on their test-beds.

\section{References}

1. K. I. Lysov, I. A. Chayuk, Operation of meliorative pumping stations, Moscow: Agropromizdat, p. 255 (1988)

2. V.F. Cheboevsky, K.P. Vishnevsky, Design of pumping stations and testing of pumping stations, Moscow:Kolos, p. 376 (2000)

3. A.K.Mikhailov, V.V. Malushenko, Vane pumps Theory calculation and design, Moscow: Mechanical Engineering, p. 283,( 1977)

4. M.Mamajonov, Pumps and pumping stations, Tashkent: Science and Technologies, p. 372, (2012)

5. G. Paresh, M. Octo, Practical Centrifugal Pumps Design, Operation ahd Maintenance, Elsevier Newnes, p. 246, (2005)

6. M.M.Muxammadiev, M.Mamajonov, T.SH. Majidov,Hydraulic Machines, Tashkent:TIIAME, p. 193, (2010)

7. G.I.Krivchenko, Hydraulic Machines: Hydraulic Turbines and Pumps, Moscow:Energoatomizdat, p. 320, (1983)

8. Z.Husain, Z. Abdullah, Z. Alimuddin, Basic Fluid Mechanics and Hydraulic Machines,(Hyderabad: BS Publications, p. 231, (2008)

9. A.A. Bulgakov, Frequency control of asynchronous electric drive, Moscow:Energoizdat, p. 216, (1982)

10. A.I. Kolesnikov, Energy saving in industrial and utility enterprises, Moscow: INFRA, p. 124, (2005) 
11. V.A. Shabanov, Methods of evaluating and ways of efficiency increase ofvariable frequency drive mainline pumps at the existing pipelines $J$ Applied academic research, 3, pp. 73-79, (2015)

12. A.I. Nadeev, N. G. Romanenko, Power saving management of electric drives of pump units, Vestnik AGTY, 2 , pp.11-15, (2011)

13. V.B. Klepikov, P.A. Korotaev, E.F. Banev, A.N. Moiseev, A. V. Timoshhenko, L. N. Sivokon, To results of the power saving electric drive operation at the second lifting station in Solonicevka town, J Energy saving, energy, energoaudit, 5, pp. 15-17, (2011)

14. V.Y. Karelin, A.V. Minaev, Pumps and pumping stations, Moscow:Strojizdat, p. 320, (1986)

15. K.A. Evtukh, E.V. Vyazunov, A.F. Barkhatov, About the economic efficiency of the replacement of pressure throttling units at the oil pumping stations by adjustable frequency-controlled actuators or hydro-clutches, Oil and Oil Products Pipeline Transportation: Science \& Technologies, 2, pp. 15-21, (2014)

16. V.A. Shabanov, E. F. Khakimov, S.F. Sharipova, The analysis of the efficiency of operating pipelines' main line pumps in case of the use of frequency converter in the function of the pressure regulator, Online scientific journal «Oil and Gas Business», 1, pp. 24-33, (2013)

17.E.V. Vyazunov, S.V. Putin, Analytical representation of characteristics of centrifugal pumps with variable rotation speed, Oil and Oil Products Pipeline Transportation: Science \& Technologies, 4, pp. 67-69, (2012)

18. M. Kozlov, A. Chistyakov, Effectiveness of implementation systems with variable frequency drives, J Modern automation technologies, 1, p. 76, (2001)

19. T. Sh. Mazhidov, E.K. Kan, A.A. Ergashev, The results of field studies of the pump unit with a frequency converter, Journal "Irrigation and Land Reclamation, 01, pp.31-34, (2016)

20. J.F. Gulich, Centrifugal Pumps, (Springer-Verlag Berlin, p. 964, (2008)

21. M.C. Sanks, G. Tchobanoglous, B.E. Bosserman, Pumping Station Design, Second Editon, Butterworth-Heinemann, p. 1067, (2010) 